\title{
The Relationship between Hip Strength and Peak Knee Valgus Angle during Single Leg Squat
}

\author{
Abdullah S. Bin Hussein ${ }^{1}$ \\ (Director of PhysicalTherapyDepartment at Prince Sultan Military Medical City - Riyadh, Kingdom of Saudi \\ Arabia)
}

\begin{abstract}
:
Objectives: Available research is limited and conflicting with regard to the role of the hipmuscles in controlling knee valgus. Thus, the aim of this study is to investigatewhether hip muscle's strength is significantly related to peak knee valgus angle (PKVA)during a single leg squat.

Methods: A correlation research design was chosen to test the hypotheses of this study in 30 healthy subjects (17 males and 13 females). Procedures included isometricstrength testing using the DILLON ED junior dynamometer for hip (abduction/adduction,flexion/extension, and internal/external rotation). Subjectswere videotaped as they performed single leg squats using two digital video cameras.Video data for squat trials were analyzed using the siliconCOACH Pro Version 6.

Results: ICC values indicated excellent reliability for PKVA(0.948). Pearson correlation coefficients demonstratedsignificant negative relationships between hip abduction $(r=-0.550)$, adduction $(r=-0.475)$, extension $(r=-0.421)$, and PKVA. Linear regression analysis revealed that hip extensors, abductors and adductorsaccounted for $17.7 \%, 30.3 \%$ and $26.6 \%$ respectively, of variance in PKVA

Conclusions: SiliconCOACH by one rater produced a reliable measurement of knee angle in frontal plane during single leg squat in healthy population. Decreased isometric strength of hip abductors, adductors and extensors was closely associated with increased peak valgus angle at the knee. This relationship is of clinical relevance when designing rehabilitative and injury prevention strengthening programs.
\end{abstract}

\section{Introduction}

Stability of the knee joint is dependent on passive restraint system (ligaments and capsule) and active neuromuscular system (muscle strength and proprioception) ${ }^{1}$.Muscle weakness can cause certain movement dysfunctions that can put certain muscles or joints in positions considered to be of high risk for injury ${ }^{2}$. Lower extremity joint stability relies on integration of various components: joint structure, non-contractile components (soft tissues), and contractile components (muscles) ${ }^{3}$. Knee valgus malalignment during dynamic tasks is a common postural dysfunction seen in the lower extremity during weight bearing activities such as landing and squatting. Knee valgus malalignment occurs when there is a simultaneous internal rotation and adduction of the femur on the tibia during closed-chain knee flexion ${ }^{4}$.Weakness or insufficient strength of muscles surrounding thigh and hip is thought to be an underlying mechanism for excessive knee valgus motion during dynamic movements ${ }^{5-8}$. While these are normal biomechanical motions, this valgus position has been theorised to be an underlying mechanism for knee injury9 .

Available research is limited and conflicting with regard to the role of the hip muscles in controlling knee valgus. Some results suggest that hip abductor musculature aids in controlling frontal plane knee motion $^{1,10,11}$, while others have reported that hip external rotation strength is more related to knee valgus movement ${ }^{12}$. It has been suggested that weak hip abductors and perhaps weak external rotators demonstrate increased knee valgus ${ }^{13}$.In contrast, no relationship was found between hip muscles and knee frontal plane control $^{14}$, or betweenhip muscle strength and peak knee valgus moment ${ }^{15}$. To-date, no investigations have reported a significant relationship between strength of hip flexors, extensors or adductors andfrontal plane position of knee during weight-bearing dynamic activities.

\section{Participants}

\section{Material And Methods}

30 subjects (17 males and 13 females) were recruited from the generalcollege population for this study. Meanage $=25.3 \pm 6.2$ years, height $=171.1 \pm 7.2 \mathrm{~cm}$, weight $=68.3 \pm 9.8 \mathrm{~kg}$. Written informed consent was obtained from allparticipants. Study was approved by the School of Healthcare Studies Ethical Research Committee, Cardiff University. 


\section{Design}

To test the hypotheses of a significant relationship between hip strength and knee kinematics in frontal plane (PKVA) during a SLS, a correlational research design was chosen. This research design is used to examine the relationship between two or more variables with no need to manipulate the independent variables ${ }^{16}$. The independent variables in this study were: isometric strength measures for hipmuscles. The dependent variable was: PKVA.

\section{Inclusion criteria:}

- No history of lower extremity injury.

- No history of low back pain or knee surgery within one year prior to the study.

\section{Exclusion Criteria:}

- Cardiovascular, pulmonary or neurological conditions that limited physical activity.

\section{Instrumentation}

Subjects were videotaped as they performed single leg squats using a digital video camera (Sony DCRTRV19E, Japan) with 1.55 Mega-pixel Advanced HAD CCDfeatures, 14-bit DXP digital signal processing, and a built-in Carl Zeiss $\mathrm{T}$ lens with 120x digital and 10x optical zoom. Video camera was placed on a tripod at a horizontaldistance of $2.5 \mathrm{~m}$, at $80 \mathrm{~cm}$ height and in front of subject, perpendicular to theplane of motion (frontal plane) to collect 2D video data. Video data for SLS trials were analysed using a custom software package called SiliconCOACH Pro Version 6 (SiliconCOACH Ltd., New Zealand). This software allows lines to be drawn on still images between anatomical markers and measures the angles formed between these lines. SiliconCOACH has been reported to have excellent reliability $(\mathrm{ICC} \geq 89$ ) for measurement of static and dynamic knee range of motion over four separate occasions in subjects unable to fully extend their knee at $90^{\circ}$ of hip flexion ${ }^{17}$.Muscle strength of individual muscle groups was measured using the Johnson Scale DILLON ED junior dynamometer (EDjr) (Dillon/Quality Plus, Inc. Camarillo, California). The DILLON ED junior dynamometer is a force measurement load sensor and digital readout in one instrument.

\section{Procedures}

This study was performed in Research Centre for Clinical Kinesiology (RCCK) at the School of Healthcare Studies, Cardiff University. All subjects participated in one session that consisted of both isometric muscle testing and single leg squats. The entire session took approximately 40 minutes. All preparations were completed before arrival of participants on data collection days. Video camera was placed on tripods at specific height from floor and distance from subject. Camera was then switched on and zoom was manually adjusted by researcher upon arrival at study venue. Then, each subject's preferred stance leg (operationally defined in this study as the preferred plant leg during kicking a ball) was determined. The preferred stance leg was selected in this study because it is the leg used to support the activities of the preferred leg by providing postural support and stability. Subjects then performed a five minute warm-up on a cycle ergometer (MonarkErgomedic $818 \mathrm{E}$, Vansbro, Sweden) in an effort to minimize the risk of physical discomfort post-testing.

Muscle strength. The force developed by each muscle group was measured in kilogram-force (kgf) using a DILLON ED junior dynamometer. The strength measures were then converted to Newton $(\mathrm{N})(1 \mathrm{kgf}$ $\left.\mathrm{x} 9.80665 \mathrm{~m} / \mathrm{s}^{2}=1 \mathrm{~N}\right)$. The chronological order of strength testing for all participants was hip flexion/extension, followed by abduction/adduction, then internal/external rotation. For each muscle group, each participant performed 2 submaximal and 2 maximal familiarization repetitions, followed by three maximal repetitions which wererecordedand then averaged to be used for analysis purposes. For each test, a five second hold for contraction and a ten second rest between contractions was applied. The point of resistance application was always perpendicular to the long axis of the limb. Tests of hip strength were conducted in standard positions ${ }^{18}$.

\section{A) Hip flexors}

While standing with back facing dynamometer and holding couch, a strap from dynamometer was applied to lower third of thigh, just above knee joint, and subjects were instructed to pull their lower limb forward and maintain an erect trunk posture (Fig 1-A).

\section{B) Hip extensors}

While standing facing dynamometer and holding pole, a strap was applied as in hip flexors test and subjects were instructed to pull their lower limb backward while maintaining an erect posture of trunk (Fig1-B).

\section{C) Hip abductors}


While standing beside dynamometer holding pole, with limb to be tested situated furthest from dynamometer, a strap was applied just above knee joint and subjects were asked to push their lower limb outward (Fig 1-C).

\section{D) Hip adductors}

While standing beside dynamometer holding pole, with limb to be tested placed next to dynamometer, a strap was applied as in abductor's testing and subjects were asked to pull their lower limb towards non-tested one (Fig 1-D).

\section{E) Hip internal rotators}

While sitting on couch with hips and knees flexed to $90^{\circ}$, with limb to be tested furthest from dynamometer, a strap running from dynamometer was applied to lower third of leg and subjects were instructed to pull their leg away in the opposite direction to non-tested leg (Fig 1-E).

\section{F) Hip external rotators}

While sitting on couch with hips and knees flexed to $90^{\circ}$, with limb to be tested next to dynamometer, a strap was applied as in internal rotators test and subjects were asked to pull their leg towards non-tested leg (Fig $1-\mathrm{F})$.

Figure1 Test positions for hip isometric muscle testing

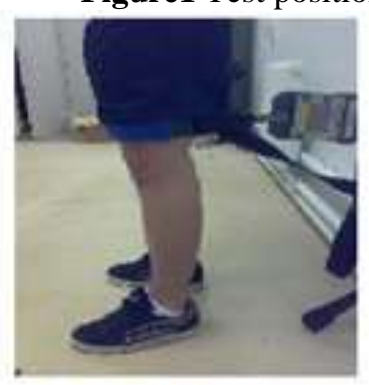

A: Hip flexors

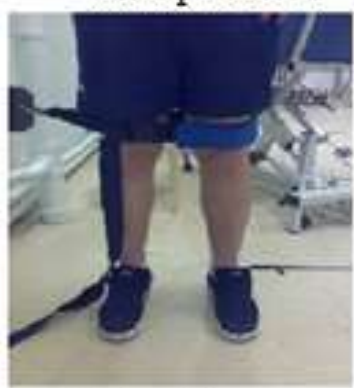

C: Hip abductors

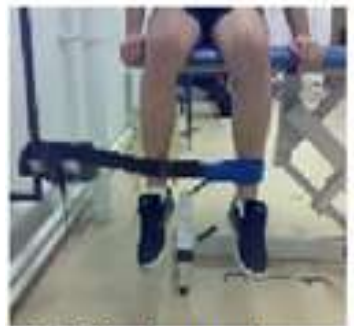

E: Hip internal rotators

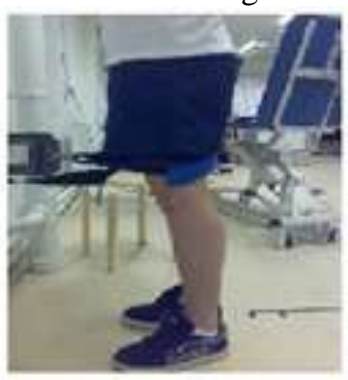

B: Hip extensors

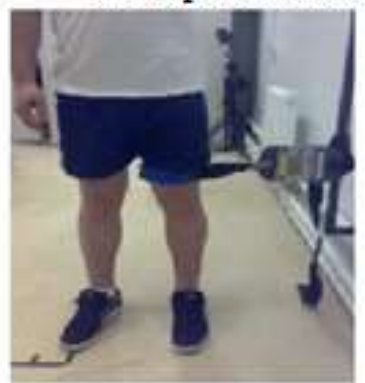

D: Hip adductors

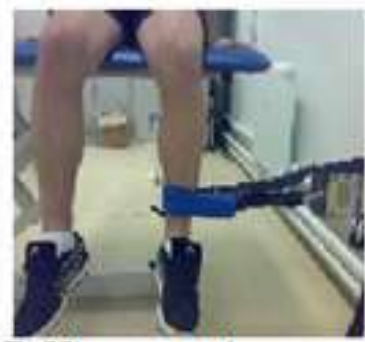

F: Hip external rotators

In order to record frontal plane motion, markers were placed on subject using sticky paper marking dots $(18 \mathrm{~mm}$ diameter) on ASIS, midpoint of knee joint (midpoint of distance between medial and lateral femoral epicondyles), and middle of ankle mortise anatomical landmarks(fig-2). The angle subtended between line formed between markers on ASIS and the midpoint of knee joint, and that formed from markers on midpoint of knee joint and middle of ankle mortise, was recorded as knee valgus angle. 
Fig-2Markers placement for single leg squat.

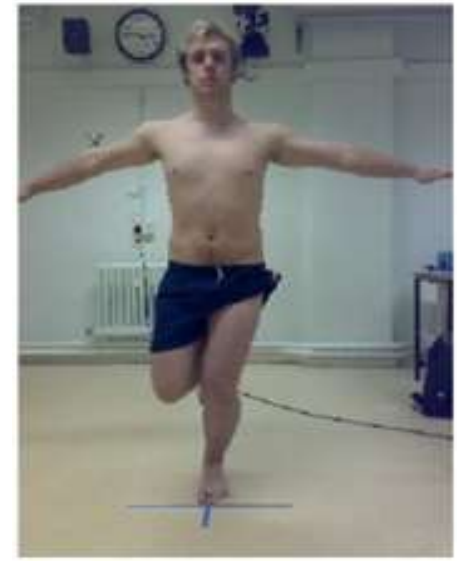

Subjects were instructed to stand on their stance limb, keep contralateral limb off floor, abduct their arms to $90^{\circ}$, squat down to $45^{\circ}$ (estimated visually) and then return to start position without losing their balance. To control degree of lower limb rotation during squat, participants were asked to place their foot on a cross mark on the floor, such that foot was pointing straight ahead. Once each subject felt comfortable with maneuver, he or she performed four to five consecutive trials. Three acceptable trials were obtained from each participant and recorded for data analysis purposes. Acceptable trials were those in which participants maintained balance and squatted to desired depth of $45^{\circ}$.

\section{Data processing - Motion analysis:}

All squat trials videotaped for each participant were transferred to a computer in School of Healthcare Studies. Each participant was assigned a file with a number. UsingSiliconCOACH Software, recorded trials for every participant were captured, and then saved on his/her file. Every participant's trials were given a specific code. Every trial in frontal plane was then reviewed in order to determine PKVA at a very slow motion. Video was run forward and backward, frame by frame, pausing at point where PKVA was estimated. At this point, zooming tool was used to zoom in on markers, then straight lines were drawn between these markers. Using normal angle tool of SiliconCOACH Program, PKVA was then measured and recorded(fig-2). PKVAs for each subject during three SLS trials were averaged for data analysis. Following collection, data wasanalyzed in Statistical Package for Social Sciences (SPSS ${ }^{\circledR}$ ) for Windows (version 15.0, SPSS Inc, Chicago, IL). PKVAs for each subject during three SLS trials were used in statistical analysis to confirm reliability of these measures when calculated by one rater (intrarater reliability) using SiliconCOACH Program.

Fig 3.Measuring peak knee valgus anglewith Silicon $\mathrm{COACH}$

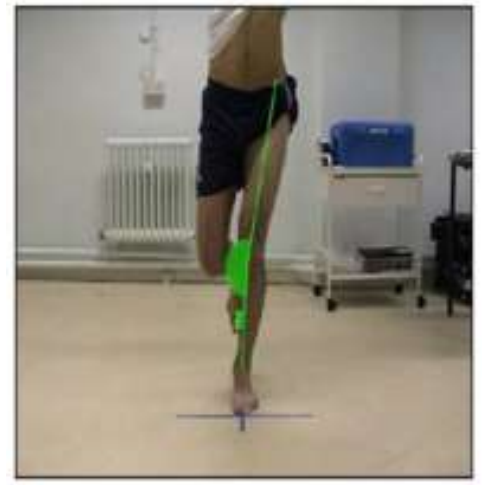

\section{Statistical Analysis}

Intrarater reliability levels were assessed using Intraclass Correlation Coefficients (ICCs). Intraclass correlations are often used as reliability coefficients for evaluation of items that are considered to be in same category or class. They compare covariance of ratings with total variance ${ }^{19}$. SPSS ${ }^{\circledR}$ version 15 provides computation of a collection of intraclass correlation coefficients.

Guidelines presented by Shrout and Fleiss ${ }^{20}$ were used for choosing appropriate form of the ICC for current study. Since the same rater performed all the measurements in this study, a two-way mixed model was used. Additionally, consistency option was selected because rater variability was considered irrelevant and 
therefore not included in denominator of estimated ICC. Selected two-way mixed model with a consistency option was equivalent to ICC $(3,1)$. For ICC output, single measure reliability was used for single measurements of rater. Classification system for ICC in this study was adopted from Landis and $\mathrm{Koch}^{21}$. Present study determined that a reliability of 0.60 or higher would be acceptable ${ }^{22}$.

Descriptive statistics including the mean, standard deviation (SD), and range of values were reported for the sample population, as well as the strength measures and knee angle. The Pearson correlation coefficient (r) was calculated to evaluate the relationship between each isometric strength measure and the dependent variable (peak knee valgus). This coefficient, also known as the product-moment correlation coefficient, measures the linear relationship between two random variables. Its value ranges from being negatively correlated (-1) to uncorrelated $(0)$ to positively correlated $(+1)^{23}$.

The SPSS ${ }^{\circledR}$ (version 15.0) statistical software was used for all correlations statistical analysis. Significance level was set at a level of 0.05 , with values of $<0.1$ accepted as a non-significant trend. The strength of correlation coefficients was interpreted according to the correlation guide documented by Guilford ${ }^{24}$.

Result: 32 subjects met inclusion criteria and agreed to participate in the study. However, two subjects demonstrated an excessive pelvis rotation during squat performance which affected resultant knee frontal-plane alignment (i.e. very large knee valgus angle). Therefore, were excluded from study. Descriptive characteristics for the subject population $(\mathrm{N}=30)$ are presented in Table 1 .

Table 1Subject characteristics

\begin{tabular}{|l|l|l|l|l|l|l|l|l|l|}
\hline & \multicolumn{3}{|l|}{ Males ( $\mathrm{n}=17)$} & \multicolumn{3}{l|}{ Females $(\mathrm{n}=13)$} & \multicolumn{2}{l|}{ Overall ( $=30)$} \\
\hline & Mean & SD & Range & Mean & SD & Range & Mean & SD & Range \\
\hline Age (yr ) & 26.9 & 5.9 & $18-38$ & 23.1 & 6.1 & $18-36$ & 25.3 & 6.2 & $18-38$ \\
\hline Height (cm) & 173.7 & 9.8 & $159-188.5$ & 167.7 & 4.6 & $158.5-173.5$ & 171.1 & 7.2 & $158.5-188.5$ \\
\hline Weight (kg) & 72.8 & 7.8 & $57.4-91.6$ & 62.4 & 6.1 & $53.8-72.4$ & 68.3 & 9.8 & $53.8-91.6$ \\
\hline
\end{tabular}

\section{Intrarater reliability}

Within session reliability was evaluated by calculating intraclass correlation coefficient (ICC) for PKVA from three trials. ICC values indicated excellent reliability for all measured variables since they were greater than $0.93^{21}$, suggesting high consistency of these measures when analysed by one rater. ICC value for PKVA was 0.948 (Table 2).

Table 2Within session intraclass correlation coefficients (ICCs) for knee kinematics.

\begin{tabular}{|c|c|c|c|c|}
\hline Angle & Mean & SD & ICC & $95 \% \mathrm{CI}$ \\
\hline Peak knee valgus & 6.61 & 5.36 & 0.948 & $0.907-0.973$ \\
\hline
\end{tabular}

Muscle strength measures:Strength values for hip flexion/extension, abduction/adduction, and internal/external rotation are listed in Table 3. These data demonstrate that male subjects had greater strength measures than female subjects for all tested muscles. Hipflexor strength was highest while lowest strength measure was that of hip internal rotator in all subjects.

Table3.Strength measures for hip and knee muscles in Newton.

\begin{tabular}{|c|l|l|l|l|l|l|l|}
\hline \multirow{2}{*}{ STRENGTH VARIABLE } & \multicolumn{2}{l|}{ MALES } & \multicolumn{2}{l|}{ FEMALES } & \multicolumn{2}{l|}{ OVERALL } \\
\cline { 2 - 8 } & Mean & SD & Mean & SD & Mean & SD \\
\hline \multirow{3}{*}{$*$} & Flexor & 269.97 & 95.03 & 201.66 & 52.83 & 240.37 & 85.57 \\
\cline { 2 - 8 } & Extensor & 262.47 & 84.11 & 205.69 & 73.66 & 237.86 & 83.47 \\
\cline { 2 - 8 } & Abductor & 253.25 & 98.31 & 164.95 & 51.44 & 214.99 & 91.69 \\
\cline { 2 - 8 } & Adductor & 261.70 & 75.88 & 192.36 & 57.33 & 231.65 & 75.90 \\
\cline { 2 - 8 } & Internal rotator & 114.80 & 46.15 & 79.96 & 26.58 & 99.70 & 42.14 \\
\cline { 2 - 8 } & External rotator & 126.91 & 42.50 & 72.67 & 15.40 & 103.41 & 42.92 \\
\hline
\end{tabular}

$\mathrm{SD}=$ Standard deviation

PKVA:Mean values of PKVA (average of three trials) for all participants during a SLS are displayed in Table 2. Positive values represent valgus positioning, whereas negative values indicate varus positioning. Individually, more than $50 \%$ of subjects had PKVAs greater than $5^{\circ}$. Majority of subjects were in a valgus knee position during a SLS, and two subjects only were in varus direction. Two subjects had greatest mean PKVA, with values exceeding $16^{\circ}$. On performing the SLS, subjects demonstrated an overall PKVA of $6.61^{\circ} \pm 5.36^{\circ}(\mathrm{mean} \pm$ $\mathrm{SD})$. When analyzed according to gender group, women exhibited a greater PKVA $\left(9.03^{\circ} \pm 5.67^{\circ}\right)$ than men 
$\left(4.76^{\circ} \pm 4.44^{\circ}\right)$.

Relationship between strength measures and PKVA:The PCCs demonstrated that the relationship between PKVAs and isometric measures of hip ranged from slight to moderate (Table4). Three relationships were moderate (hip abduction, adduction, and extension), highest observed was hip abduction strength at $\mathrm{r}=0.550$. Two relationships were low (hip flexion and external rotation) and one was slight (hip internal rotation).

Table4. PCCs(r) and p values of the relationships between hip muscle strength and peak knee valgus angle.

\begin{tabular}{|c|c|c|c|}
\hline \multicolumn{4}{|c|}{ Peak knee valgus angle } \\
\hline $\mathbf{r}$ & p & & \\
\hline \multirow{6}{*}{ 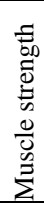 } & Hip flexor & $-0.356 \mathrm{~F}$ & 0.054 \\
\hline & Hip extensor & $-0.421 *$ & 0.021 \\
\hline & Hip abductor & $-0.550 *$ & 0.002 \\
\hline & Hip adductor & $-0.475 *$ & 0.008 \\
\hline & Hip internal rotator & -0.169 & 0.371 \\
\hline & Hip external rotator & -0.206 & 0.275 \\
\hline
\end{tabular}

$\mathrm{r}=$ Pearson correlation coefficient

* Denotes significance at $\mathrm{p}=0.05$

干 Denotes a non-significant trend $(\mathrm{p}<0.1)$

Statistical analysis of PCC (r) revealed significant negative relationships between four strength measures and PKVA, with knee valgus angles increasing as strength decreased (Table 3). Significant negative correlations were found between hip abduction $(\mathrm{r}=-0.550, \mathrm{p}=0.002)$, extension $(\mathrm{r}=-0.421, \mathrm{p}=0.021)$, adduction $(\mathrm{r}=-0.475, \mathrm{p}=0.008)$ and PKVA. However, there were no significant relationships between strength measures of hip flexion, internal rotation, or external rotation and PKVA $(p>0.05)$, although hip flexion showed a nonsignificant trend towards a small relationship.

Linear regression analysis revealed that three strength variables were significantly associated with PKVA $(\mathrm{p}<0.05)$. These three strength variables were hip extensor, abductor and adductor which accounted for $17.7 \%, 30.3 \%$, and $26.6 \%$ respectively, of variance in PKVA (Table 5).

Table 6 presents quantification of relationship between PKVA and three muscle's strength. With every increase of one unit in hip extensor, abductor and adductor strength, PKVA (on average) decreased by 0.027 , 0.032 , and 0.034 units, respectively $(\mathrm{p}<0.05)$.

Table 5Linear regression results (dependent variable=PKVA)

\begin{tabular}{|c|c|c|c|c|c|}
\hline & \multicolumn{5}{|c|}{ PKVA } \\
\hline & $\mathbf{r}$ & $\mathbf{r}^{2}$ & $\mathbf{F}$ & Significance & SEE \\
\hline Hip Extensor & -0.421 & 0.177 & 6.017 & 0.021 & 4.95 \\
\hline Hip Abductor & -0.550 & 0.303 & 12.172 & 0.002 & 4.56 \\
\hline Hip Adductor & -0.475 & 0.226 & 8.161 & 0.008 & 4.80 \\
\hline
\end{tabular}

Table 6Regression coefficients.

\begin{tabular}{|l|l|l|l|l|l|}
\hline & \multirow{2}{*}{$\mathrm{a}$} & \multirow{2}{*}{$\mathrm{b}$} & \multirow{2}{*}{ Significance } & \multicolumn{2}{|l|}{ 95\% CI for b } \\
\cline { 5 - 6 } & & & & \multicolumn{2}{|l|}{ LowerUpper } \\
\hline Hip Extensor & 13.042 & -0.027 & 0.021 & -0.050 & -0.004 \\
\hline Hip Abductor & 13.536 & -0.032 & 0.002 & -0.051 & -0.013 \\
\hline Hip Adductor & 14.392 & -0.034 & 0.008 & -0.058 & -0.010 \\
\hline
\end{tabular}

a: Intercept (value of PKVA when strength $=0$ ).

b: Slope (change in PKVA that corresponds to a one-unit change in strength).

CI: Confidence interval.

\section{Discussion}

This study was conducted to examine whether a significant relationship exists between hip strength and PKVA during a SLS.Intrarater reliability- ICC values for three trials analysed in one session were high, implying high consistency of knee measures (PKVAs). These results also suggest that individual's present study had minimal variability between three trials, implying that they squatted indifferently on consecutive trials.

Overall,results of study are promising and findings support use of SiliconCOACH Software in measuring knee angle during dynamic activities, such as squat. However, it must be noted that this software greatly relies on clear appearance of markers. Therefore, markers should be kept visible throughout the videotaping process. 
This study hypothesized that hip abduction strength would be significantly related to the PKVA when performing a SLS. Study results revealed a statistically significant relationship existed between hipabduction strength and PKVA during a SLS in a healthy population. This relationship suggests that subjects with greater hip abduction strength may have better control of knee in frontal plane and therefore be likely to demonstrate less valgus angle values. Hip abduction strength has been reported to play a critical role in dynamic stability of lower extremity in frontal plane during weight-bearing activities ${ }^{1,4,25}$. In weight-bearing activities, while gluteus medius is primary hip abductor, its intermediate and posterior fibres act as both abductors and external rotators of hip when hip is flexed less than $20^{\circ} .{ }^{26}$ It is therefore reasonable to presume that weakness in gluteus medius and, to a lesser extent, gluteus maximus, will lead to decreased control of thigh abduction and external rotation.

As a result, thigh will tend to move into increased adduction and internal rotation which may place knee in an increased valgus position. ${ }^{4}$ Results derived from present study concur with those reported in some studies and differ from those reported in other studies. For example, correlation coefficient between hip abduction strength and PKVA in present study was consistent with that reported by Claiborne ${ }^{4}$, who found a relatively small but significant correlation coefficient between isokinetic strength of hip abduction and frontal plane knee motion (peak knee valgus minus standing frontal plane knee position) during a SLS. Jacobs and Mattacola reported a significant negative relationship between female peak eccentric strength and PKVA during jump landing tasks. However, they did not report the overall correlation magnitude; thus, comparing their study finding with that of the current study would be inappropriate ${ }^{27}$.Current study's finding also contrasts with that reported in some studies that found a small overall correlation between hip abductor strength and knee valgus.

Independent of gender, Willson et al. reported a non-significant relationship between hip abduction isometric strength and 2D knee frontal plane projection angle during a SLS ${ }^{12}$.Similarly, there was nonsignificant overall relationship between isometric hip abduction strength and kneeabduction during jump landings ${ }^{10}$. Linear regression results indicated that decreased PKVA is associated with a significant increase in hip abduction strength, of about 0.032 units for every one-unit increase in average strength. Also, variance in hip abduction strength accounted for $30 \%$ of the variance in PKVA. This value is much higher than hip abduction strength accounted for $13 \%$ of variance in frontal plane motion of knee during a SLS ${ }^{1}$. Hip abduction in current study can be interpreted as a large clinical effect. Certainly, it reflects close relationship between PKVA and hip abduction strength. It also indicates that isometric strength of hip abduction can be used as a significant predictor when attempting to calculate PKVAduring a SLS ${ }^{28}$. This study also hypothesized that a significant relationship would exist between hip extension strength and PKVA. Study results provided evidence of a statistically significant relationship between hip extension strength and PKVA during a SLS.The results concur with Clark who suggested that individuals who demonstrate knee valgus have weak gluteus maximus control during squatting ${ }^{2}$.Gluteus maximus acts both as a prime hip extensor and as a stabiliser of the hip and sacroiliac joints during activities such as squatting ${ }^{29}$.

The present study's findings also offer a basis for practical applications in injury prevention. They suggest that it is possible to develop more effective prevention strategies, targeting improvement of muscle support to dynamic knee stability in the frontal plane, to reduce knee injury rates. However, the design of the current study does not allow assertion that hip strengthening will be beneficial for preventinglower extremity injuries, although strength training has been shown to decrease the incidence of serious knee injury in the highrisk female sports population ${ }^{30}$.

Present study recruited only recreationally active subjects without lower extremity problems; therefore, it is not possible to generalize the results obtained to athletic or patient populations. Also, the level and type of physical activity of subjects was not taken into account. It is possible that some subjects may have had more experience with a SLS task or similar tasks to the methods used in the current study.

Lastly, the current study provides additional support for a simple method to assess kneefrontal plane alignment during weight-bearing activity using instruments and procedures suitable for a clinical setting. The testing procedures in the present study were designed to be relatively easy to perform by researchers, trainers, and therapists, and portable equipment was used so that it could be operated outside a formal laboratory. The testing procedures included videotaping a subject while performing a simple task, namely, a SLS, and then measuring the knee frontal plane angle using a 2D motion analysis program, namely, Silicon $\mathrm{COACH}$.

\section{Conclusion}

Using the Silicon COACH by one rater produced a reliable measurement of knee anglesin frontal plane during a single leg squat in a healthy population. Decreased isometric strength of the hip abductors, adductors and extensors was closely associated with increased peak valgus angle at the knee. This relationship is of clinical relevance when designing rehabilitative and injury prevention strengthening programs. 


\section{References}

[1]. Claiborne, T.L., Armstrong, C.W. et al. Relationship between hip and knee strength and knee valgus during a single leg squat. Journal of Applied Biomechanics2006, 22(1), 41-50.

[2]. Clark, M.A. Core stabilization training in rehabilitation. In: Prentice, W.E., Voight, M.L. eds. Techniques in Musculoskeletal Rehabilitation. New York, NY: McGraw-Hill 2001, 259-278.

[3]. Riemann, B.L. and Lephart, S.M. (2002) The sensorimotor system, Part I: The

[4]. physiologic basis of functional joint stability. Journal of Athletic Training 2002, 37, 71-79.

[5]. Zeller, B., McCrory, J. et al. Differences in kinematics and electromyographic activity between men and women during the singlelegged squat. American Journal of Sports Medicine 2003, 31,449-456.

[6]. Powers, C.M. The influence of altered lower-extremity kinematics on patellofemoral joint dysfunction: A theoretical perspective. Journal of Orthopedic Sport Physical Therapy 2003, 33, 693-646.

[7]. Fredericson, M., Cookingham, C.L. et al. (2000) Hip abductor weakness in distance runners with iliotibial band syndrome. Clinical Journal of Sport Medicine, 10,169-175.

[8]. Ireland, M.L., Willson, J.D. et al. Hip strength in females with and without patellofemoral pain. Journal of Orthopedic Sport Physical Therapy 2003, 33,671-676.

[9]. Hewett, T.E., Stroupe, A.L. et al. Plyometric training in female athletes. Decreased impact forces and increased hamstring torques. American Journal of Sports Medicine 1996, 24, 765-773.

[10]. Ireland, M. Anterior cruciate ligament injury in female athletes: epidemiology. Journal of Athletic Training 1999, 34, 150-154.

[11]. Jacobs, C.A., Uhl, T.L. et al. Hip abductor function and lower extremity landing kinemtaics: sex differences. Journal of Athletic Training 2007, 42, 76-83.

[12]. Hollman, J. H., Ginos, B. E., Kozuchowski, J., Vaughn, A. S., Krause, D. A., \&Youdas, J. W. Relationships between knee valgus, hip-muscle strength, and hip-muscle recruitment during a single-limb step-down. Journal of sport rehabilitation 2009, $18(1), 104$.

[13]. Willson, J.D., Ireland, M.L., Davis, I. Core strength and lower extremity alignment during single leg squats. Medicine and Science in Sports and Exercise 2006, 38(5), 945-952.

[14]. Cashman, G. E., The effect of weak hip abductors or external rotators on knee valgus kinematics in healthy subjects: a systematic review. Journal of sport rehabilitation 2012, 21(3), 273-284

[15]. Thijs, Y., Van Tiggelen, D. et al. Relationship between hip strength and frontal plane posture of the knee during a forward lunge. British Journal of Sports Medicine 2007,41, 723-727.

[16]. Bencke, J., Lauridsen, H. B., Thorborg, K., Hölmich, P., Andersen, L. L., Aagaard, P., \&Zebis, M. K. The influence of hip joint control on knee joint valgus moment in young female elite athletes. British journal of sports medicine 2014, 48(7), 566-566.

[17]. Hicks, C. Research Methods for Clinical Therapists 2004. 4th ed. Edinburgh: Churchill Livingstone.

[18]. Cronin, J., Nash, M. et al. Assessing dynamic knee joint range of motion usingsiliconcoach. Physical Therapy in Sport 2006, 7, 191194.

[19]. Fallatah, K. The relationship between lower limb muscles' strength and mobility patients with myotonic dystrophy 2005. MSc thesis. Cardiff University.

[20]. Yaffee, R. A. (1998) Enhancement of reliability analysis: Application of intraclass correlations with SPSS/Windows v.8. [WWW] < URL:http://www.nyu.edu/its/socsci/Docs/intracls.html> [Accessed 16 April 2008]

[21]. Shrout, P.E. and Fleiss, J.L. Intraclass correlations: uses in assessing rater . Psychological Bulletin 1979, 86, $420-428$.

[22]. Landis, J.R. and Koch, G.G. The measurement of observer agreement for categorical data. Biometrics 1977, 33, 159-174

[23]. Walter, D.S., Eliasziw, M. et al. Sample size and optimal design for reliability studies. Statistics in Medicine 1998, 17, 101-10.

[24]. Zou, K. H., Tuncali, K. et al.-Correlation and simple linear regression. Radiology 2003,227, 617-628.

[25]. Guilford, J.P. Fundamental Statistics in Psychology and Education 1956. (p. 145) New York: McGraw Hill.

[26]. Powers, C.M. The influence of altered lower-extremity kinematics on patellofemoral joint dysfunction: A theoretical perspective. Journal of Orthopedic Sport Physical Therapy 2003, 33, 693-646.

[27]. Delp, S. L., Hess, W.E. et al. Variation of rotation moment arms with hip flexion. Journal of Biomechanics 1999, 32, 493-501.

[28]. Jacobs, C. and Mattacola, C. Sex differences in eccentric hip-abductor strength and knee-joint kinematics when landing from a jump. Journal of Sport Rehabilitation 2005, 14,346-355

[29]. Cohen, J.A power primer. Psychological Bulletin 1992, 112(1) 155-159.

[30]. Isear, J. A., Jr, Erickson, J. C. et al. (1997) EMG analysis of lower extremity muscle recruitment patterns during an unloaded squat. Medicine and Science in Sports and Exercise, 29, 532-539.

[31]. Hewett, T., Lindenfeld, T. et al. The effect of neuromuscular training on the incidence of knee injury in female athletes: a prospective study. American Journal of Sports Medicine 1999, 27,699-706. 